\title{
Expression and oncogenic properties of membranous Notch1 in oral leukoplakia and oral squamous cell carcinoma
}

\author{
XU DING ${ }^{1,2^{*}}$, YANG ZHENG $^{1,2^{*}}$, ZHAO WANG $^{1,7}$, WEI ZHANG $^{1}$, YIBO DONG $^{1,2}$, WANTAO CHEN $^{3,4}$,

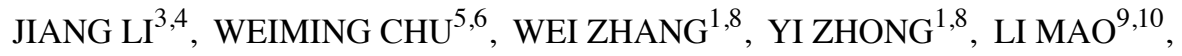 \\ XIAOMENG SONG ${ }^{1,2}$ and YUNONG WU ${ }^{1,2}$
}

\begin{abstract}
${ }^{1}$ Jiangsu Key Laboratory of Oral Diseases, Nanjing Medical University; ${ }^{2}$ Department of Oral and Maxillofacial Surgery, Affiliated Hospital of Stomatology, Nanjing Medical University, Nanjing, Jiangsu 210029;

${ }^{3}$ Department of Oral and Maxillofacial Surgery, Ninth People's Hospital; ${ }^{4}$ Shanghai Key Laboratory of Stomatology, Shanghai Jiao Tong University School of Medicine, Shanghai 200011; ${ }^{5}$ Department of Stomatology,

Northern Jiangsu People's Hospital; ${ }^{6}$ Clinical Medical School, Yangzhou University, Yangzhou, Jiangsu 225001,

P.R. China; ${ }^{7}$ Australian Institute for Bioengineering and Nanotechnology, The University of Queensland, Brisbane, QLD 4072 Australia; ${ }^{8}$ Department of Oral Pathology, Affiliated Hospital of Stomatology, Nanjing Medical University,

Nanjing, Jiangsu 210029, P.R. China; ${ }^{9}$ Department of Oncology and Diagnostic Sciences,

University of Maryland School of Dentistry, University of Maryland;

${ }^{10}$ Marlene and Stewart Greenebaum Cancer Center, University of Maryland, Baltimore, MD 21201, USA
\end{abstract}

Received September 12, 2017; Accepted March 16, 2018

DOI: $10.3892 /$ or.2018.6335

\begin{abstract}
Notch1 signaling is essential for tissue development and tumor progression. This signaling pathway has also been implicated in oral leukoplakia (OL) and oral squamous cell carcinoma (OSCC). However, the role of Notch1 expression in $\mathrm{OL}$ and its malignant transformation is unknown. This study aimed to examine the Notch1 expression patterns by immunohistochemistry (IHC) in a cohort of 78 Chinese patients with $\mathrm{OL}$ and to analyze the relationship between the patterns and progression of OL to OSCC. Strong Notch1 staining was observed in $10(13 \%)$ of the 78 OL patients, but it was not associated with any of the clinicopathological parameters. However, we observed membranous Notch1 expression in 24 (31\%) of the OL samples. Membranous Notch1 expression was significantly associated with the severity of dysplasia $(\mathrm{P}<0.001)$ and development of OSCC $(\mathrm{P}=0.003)$. By multivariate analysis, membranous Notch1 expression was found to be the only independent factor for OSCC development in the patient population $(\mathrm{P}=0.019)$. Among the 24 patients with
\end{abstract}

Correspondence to: Professor Yunong $\mathrm{Wu}$ or Professor Xiaomeng Song, Jiangsu Key Laboratory of Oral Diseases, Nanjing Medical University, 136 Hanzhong Road, Gulou, Nanjing, Jiangsu 210029, P.R. China

E-mail: yunongwu@aliyun.com

E-mail: xiaomengsong@njmu.edu.cn

Key words: oral squamous cell carcinoma, oral leukoplakia, immunohistochemistry, membrane Notch1, OSCC-free survival, 4-NQO membranous Notch1 expression, $11(46 \%)$ developed OSCC compared to $8(15 \%)$ of the 54 patients without such expression $(\mathrm{P}=0.001$, determined by log-rank test). Furthermore, we established a 4-nitroquinoline-1-oxide (4NQO)-induced murine OSCC model and studied the Notch1 expression patterns in different stages of carcinogenesis. We observed that the extent of expression of membranous Notch1 increased during carcinogenesis. These data indicated a relationship between membranous Notch1 expression and OSCC risk in patients with OL and suggested that membranous Notch1 served as a biomarker for assessing OSCC risk.

\section{Introduction}

Oral leukoplakia (OL) is a white patch in the oral cavity which cannot be classified as any other known disease (1). It is the most common oral precancerous lesion with high risk to transform into oral squamous cell carcinoma (OSCC) (2-4). A number of etiological factors have been implicated in the onset and transformation of OL, such as tobacco, alcohol, areca nut chewing and chronic mechanical injuries (5). However, no apparent etiological factor could be found in some OL patients, suggesting susceptibility plays a role in the development of OL and OSCC. Clinically, severe dysplasia is an important indicator for OSCC risk in patients with OL whereas OL with mild dysplasia or even no dysplasia can also transform into OSCC $(2,4)$. Previously, we revealed that protein expression, such as podoplanin expression, may serve as a biomarker to assess OSCC risk in patients with OL $(6,7)$. However, the roles of some proteins may depend on cell types and stages during tumorigenesis. For example, Smad4 may function as a tumor suppressor in OSCC development as evidenced in mouse models (8). However, we revealed that high Smad4 levels in 
OL were associated with OSCC development, suggesting a complex role of Smad4 in oral tumorigenesis (9).

The Notch signaling pathway is highly conserved during evolution. In mammals, there are five Notch ligands (Jagged1, Jagged2, DLL1, DLL3 and DLL4), and four Notch receptors (Notch1-4). Upon ligand binding, the receptors are cleaved at the transmembrane domain by the $\gamma$-secretase complex, which releases the active Notch intracellular domain (NICD). NICD enters the nucleus to form a transcription complex with CSL and mastermind-like proteins, leading to transactivation of Notch target genes (10-12).

The role of Notch1 signaling in cancer is, however, cell context dependent (13). Activated Notch1 signaling has been shown to inhibit the growth of hepatocellular carcinoma, small-cell lung cancer, and prostate cancer cells $(14,15)$. In cervical cancers, Notch1 is considered to be a tumor suppressor. It can activate p53, a tumor suppressor, leading to cell cycle arrest and apoptosis (16). However, overexpression of Notch1 has been observed in cutaneous SCC, suggesting Notch1 signaling may be activated in some SCCs (17). In OSCC, while overexpression of Notch1 has been reported in some studies (18-21), downregulation of Notch1 has also been observed (22). Notably, inactivating mutations of Notch1 are detected in 10-15\% of head and neck squamous cell carcinoma (HNSCC) including OSCC samples of Caucasian patients. However, Notch1 mutations are detected in $\sim 40 \%$ of OSCC samples from Chinese patients including a considerable fraction of potentially activating mutations (23-25). Recently, Izumchenko et al (26), reported a similar Notch1 mutation rate in a Chinese OSCC cohort as well as a high Notch1 mutation rate in a Chinese OL cohort. These results revealed that the Notch1 pathway plays a pivotal role in early oral tumorigenesis, particularly in the Chinese population. To further explore the role of Notch1 in OL and its progression to OSCC, we examined Notch1 expression patterns in OL and analyzed relationships between the expression patterns and clinicopathological parameters as well as OSCC progression in a Chinese OL cohort.

\section{Materials and methods}

Patients and tissue samples. Seventy-eight tissue samples from Chinese patients with pathologically confirmed OL between January 1996 and December 2010 were obtained from the Department of Pathology, School of Stomatology, Shanghai Jiao Tong University. Nineteen of the $78 \mathrm{OL}$ patients later developed OSCC. Tumor tissues from the 19 patients were also available for the study. The diagnosis of all the samples was verified by examining a hematoxylin and eosin section from each tissue block used in the study. Clinicopathological parameters and the follow-up information were obtained through chart review. Informed consent was received from each enrolled patient, and the research was carried out with the approval from the Ethics Committee of Shanghai Jiao Tong University (Shanghai, China).

Cell line and reagents. The WSU-HN4 cell line previously described (27) was cultured in Dulbecco's modified Eagle's medium (DMEM; Gibco-BRL, Grand Island, NY, USA) supplemented with $10 \%$ heat-inactivated fetal bovine serum (FBS; Gibco-BRL) at $37^{\circ} \mathrm{C}$ in a humidified $5 \% \mathrm{CO}_{2}$ atmosphere. For the EDTA (Sigma-Aldrich; Merck KGaA, Taufkirchen, Bayern, Germany) activation assay, WSU-HN4 cells were washed twice in phosphate-buffered saline (PBS) and incubated in PBS, $2.5 \mathrm{mM}$ EDTA or $2.5 \mathrm{mM} \mathrm{CaCl} \mathrm{C}_{2}$ (Sigma-Aldrich; Merck KGaA) for $15 \mathrm{~min}$ at $37^{\circ} \mathrm{C}$. The cells were then recovered in DMEM for $4 \mathrm{~h}$ and subjected to western blot analysis or immunofluorescence staining.

Western blot analysis and immunofluorescence staining. Extracted proteins from cells were assessed using the BCA protein assay reagent kit (Pierce; Thermo Fisher Scientific Inc., Waltham, MA, USA). Protein samples were resolved by $10 \%$ SDS-PAGE and immunoblotted with rabbit anti-Notch1 $\mathrm{XP}^{\text {тм }}$ (1:1,000 dilution; clone D1E11; cat. no. 3608; Cell Signaling Technology, Inc., Danvers, MA, USA) and appropriate HRP-conjugated antibodies anti-rabbit and anti-mouse IgG HRP-linked secondary antibodies (1:2,000 dilution; cat. no. 7074; cat. no. 7076; Cell Signaling Technology, Inc). Mouse anti- $\beta$-actin ( $\beta$-actin; clone AC-74; Sigma-Aldrich; Merck KGaA) was used to normalize protein loading. For immunofluorescence staining, the cells were washed twice in PBS and fixed with $10 \%$ formalin in PBS for $10 \mathrm{~min}$. The cells were then treated with $0.25 \%$ Triton X-100 (Sigma-Aldrich; Merck KGaA) for $15 \mathrm{~min}$ and were blocked with $2.5 \%$ normal goat serum in PBS for $40 \mathrm{~min}$. The first antibody (1:500 dilution) was the same as that used previously for the western blotting. The second antibody was goat fluorescein-conjugated anti-rabbit IgG (1:500; DI-1488; Vector Laboratories, Inc., Burlingame, CA, USA). Slides were mounted with Vectashield containing DAPI (Vector Laboratories, Inc.).

Immunohistochemistry. Briefly, 4- $\mu \mathrm{m}$ formalin-fixed, paraffin-embedded tissue sections were de-paraffinized and rehydrated. Heat-mediated antigen retrieval using $0.01 \mathrm{M}$ sodium citrate buffer ( $\mathrm{pH}$ 6.0) was performed and endogenous peroxidase was quenched with $3 \%$ hydrogen peroxide for $20 \mathrm{~min}$ at room temperature. After incubation with 5\% normal goat serum to reduce nonspecific binding, the sections were incubated with rabbit anti-Notch1 (D1E11) XP ${ }^{\text {TM }}$ (1:500 dilution; clone D1E11; cat. no. 3608; Cell Signaling Technology, Inc.) according to the manufacturer's protocol.

Notch1 immunoreactivity in both animal and human samples was semi-quantitatively evaluated according to staining intensity and distribution using the immunoreactive score. The grading for the percentage of positive cells was as follows: 0 , negative; $1,<10 \% ; 2,11-50 \% ; 3,51-80 \%$; and $4,>80 \%$ positive cells. For tinctorial strength, scores were distributed as: no staining, 0 points; light yellow, 1 point; brownish yellow, 2 points; dark brownish yellow, 3 points. Finally, the result was classified into four grades by multiplying the two scores aforementioned: 0 points, negative $(-)$; $1-4$ points, weakly positive $(+)$; $5-8$ points, positive $(++)$; 9-12 points, strongly positive $(+++)$. Scores for $>5$ points were regarded as positive $(28,29)$.

Animal model and treatments. A total of 48-week-old male SD rats were purchased from the Nanjing Medical University Animal Research Center. The animal experiments were approved by the Animal Ethics Committee and all the 

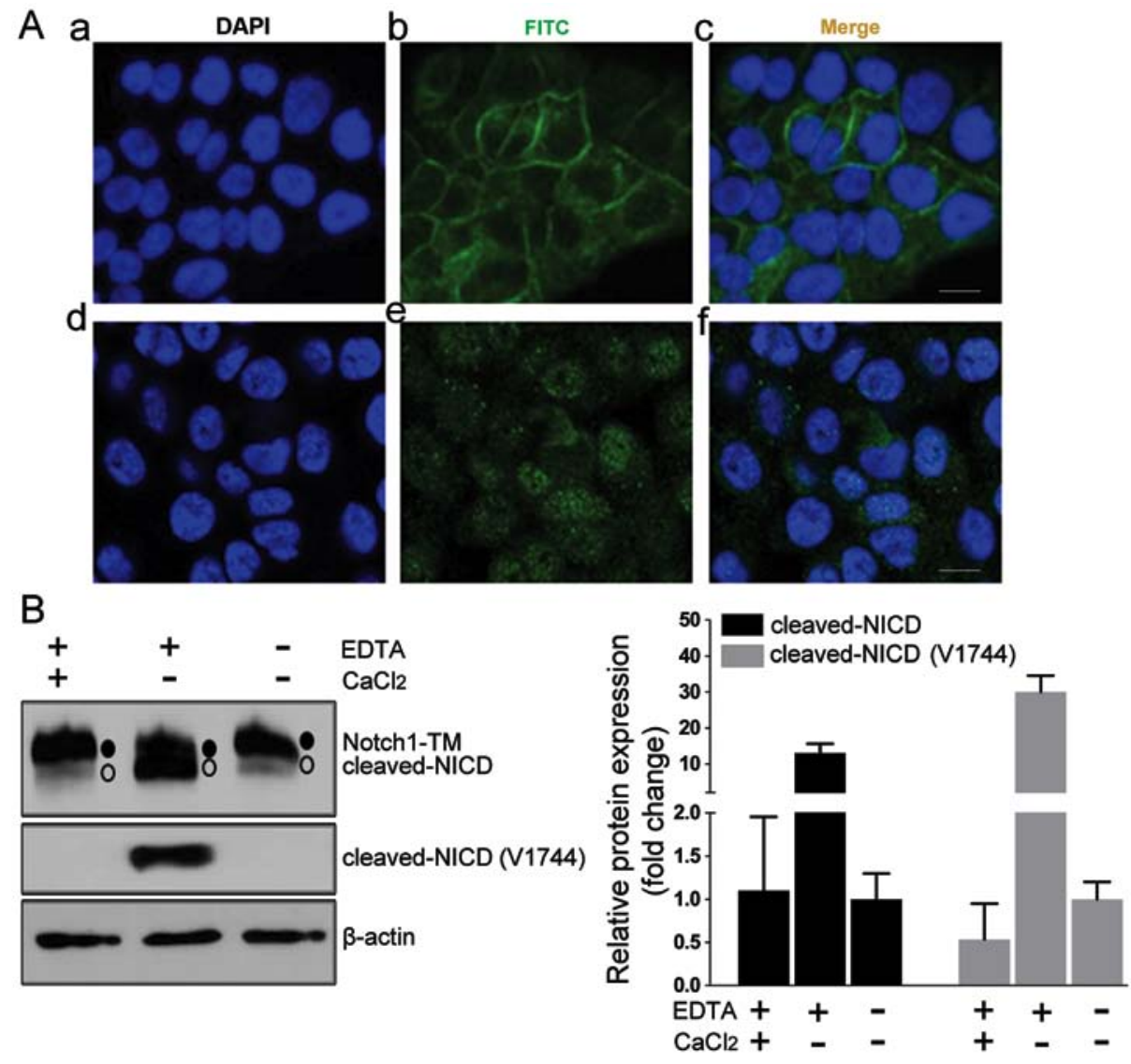

Figure 1. Validation of Notch1 antibody for detection of membranous and nuclear Notch1 in HN4 cells. (A) Immunofluorescent staining was observed after HN4 cells were treated with PBS (a-c) or $2.5 \mathrm{mM}$ EDTA (d-f) for $10 \mathrm{~min}$ and immunostained with the anti-Notch1 antibody. The cells treated with PBS demonstrated strong membranous staining of Notch1 (a-c). An obvious nuclear enrichment of Notch1 staining was observed in the cells treated with EDTA (d-f). Scale bars, $10 \mu \mathrm{m}$. (B) Western blot analysis (left) and the quantification (right) of protein extracted from HN4 cells with different treatments revealed transmembranous (TM) Notch1 (S2 cleaved and S3 uncleaved, solid dot) and activated NICD (S3 cleaved, hollow circle). In PBS-treated HN4 cells, the Notch1 protein was mostly in the transmembranous form. The EDTA treatment induced NICD S3-cleaved, as a smaller size band was detected by the Notch1 antibody, and the cleaved state was further confirmed by the Notch1 Val1744-specific antibody. The treatment with $\mathrm{CaCl}_{2}$ neutralized the function of EDTA and reversed the S3-cleaved status induced by EDTA. The PBS-treated cells were set as the control group. The quantification analysis was calculated from three independent experiments.

procedures were performed following the institutional animal welfare guidelines. After a week of acclimation, the rats were separated into three experimental groups (Groups 1,2 and 3, $\mathrm{n}=10$ for each group) and one group for control (Group 4, $\mathrm{n}=10$ ) randomly in separate cages. The rats in the experimental groups were treated with $40 \mu \mathrm{g} / \mathrm{ml}$ 4-NQO (Sigma-Aldrich; Merck KGaA) in the drinking water, while the rats in the control group were treated with an equivalent volume of propylene glycol in water $(30,31)$. The treatment lasted for 8 weeks (Group 1), 16 weeks (Group 2) or 24 weeks (Groups 3 and 4). Then, the rats were euthanized, and the tongues were resected and immediately fixed in 5\% paraformaldehyde solution for 12-24 $\mathrm{h}$ and paraffin-embedded for further analysis.

Statistical analysis. Statistical analysis was performed using SPSS 17.0 (SPSS, Inc., Chicago, IL, USA). The Chi-square or Fisher's exact tests were used to determine the relationship between the expression of Notch1 and clinicopathological parameters. OSCC-free survival (OFS) was determined as the outcome variable. The association between Notch1 expression and OFS was estimated using the log-rank test. All the variables were subjected to a univariate and multivariate analysis using Cox proportional hazards regression model. The hazard ratios
(HRs) with their corresponding 95\% confidence intervals (CIs) and P-values were reported. All the tests were two sided and a P-value $<0.05$ was considered as statistically significant.

\section{Results}

Clinicopathological characteristics of patients and ascertainment of Notch1 expression. Of the 78 patients with OL, 33 (42\%) were male and $45(58 \%)$ were female with age ranging from 27 to 85 years old (mean, 56 years old). Forty-nine (63\%) of the OL lesions were located on the tongue and 29 (37\%) at other anatomic locations in the oral cavity. During the median 74.18 months follow-up period, 19 of the 78 (24\%) patients developed OSCC. The clinicopathological characteristics are listed in Table I. No statistically significant difference was observed in age, sex, OL location, smoking and alcohol consumption statuses between those who developed OSCC and those who did not, except for a trend towards more OL with severe dysplasia in the group that developed OSCC $(\mathrm{P}=0.063$, Chi-square test, Table I).

To ensure the quality of the Notch1 detection method, we first assessed the anti-Notch1 antibody by immunofluorescence in HN4 cells (Fig. 1A-a-c). Then, we artificially induced 
Table I. Correlation between clinicopathological features and malignant transformation (OSCC).

\begin{tabular}{|c|c|c|c|c|c|c|c|}
\hline & \multirow[b]{2}{*}{ No. } & \multirow[b]{2}{*}{$(\%)$} & \multicolumn{2}{|c|}{ MT } & \multicolumn{2}{|c|}{ UT } & \multirow[b]{2}{*}{ P-value } \\
\hline & & & $\mathrm{n}$ & $(\%)$ & $\mathrm{n}$ & $(\%)$ & \\
\hline All patients & 78 & 100 & 19 & 24 & 59 & 76 & \\
\hline \multicolumn{8}{|l|}{ Age (years) } \\
\hline$\geq 60$ & 24 & 31 & 7 & 9 & 17 & 22 & 0.51 \\
\hline$<60$ & 54 & 69 & 12 & 15 & 42 & 54 & \\
\hline \multicolumn{8}{|l|}{ Sex } \\
\hline Male & 33 & 42 & 5 & 6 & 28 & 36 & 0.105 \\
\hline Female & 45 & 58 & 14 & 18 & 31 & 40 & \\
\hline \multicolumn{8}{|l|}{ Grade of dysplasia } \\
\hline Mild-moderate & 59 & 76 & 11 & 14 & 48 & 62 & 0.063 \\
\hline Severe & 19 & 24 & 8 & 10 & 11 & 14 & \\
\hline \multicolumn{8}{|l|}{ Lesion site } \\
\hline Non-tongue & 49 & 63 & 10 & 13 & 39 & 50 & 0.297 \\
\hline Tongue & 29 & 37 & 9 & 11 & 20 & 26 & \\
\hline \multicolumn{8}{|l|}{ Smoking } \\
\hline Never & 57 & 73 & 16 & 21 & 41 & 53 & 0.327 \\
\hline Past and present & 15 & 19 & 2 & 3 & 13 & 17 & \\
\hline Unknown & 6 & 8 & 1 & 1 & 5 & 6 & \\
\hline \multicolumn{8}{|l|}{ Alcohol intake } \\
\hline Never & 58 & 74 & 15 & 19 & 43 & 55 & 0.748 \\
\hline Past and present & 15 & 19 & $\mathbf{3}$ & 4 & 12 & 15 & \\
\hline Unknown & 5 & 6 & 1 & 1 & 4 & 5 & \\
\hline \multicolumn{8}{|l|}{ Notch1 expression } \\
\hline Strong & 10 & 13 & 4 & 5 & 6 & 8 & 0.246 \\
\hline Weak to moderate & 68 & 87 & 15 & 19 & 53 & 68 & \\
\hline \multicolumn{8}{|l|}{ MN } \\
\hline Positive & 24 & 31 & 11 & 14 & 13 & 17 & 0.003 \\
\hline Negative & 54 & 69 & 8 & 10 & 46 & 59 & \\
\hline
\end{tabular}

MT, malignant-transformed oral leukoplakia; UT, untransformed oral leukoplakia; MN, membrane Notch1 expression.

the dissociation of the heterodimer by treating the cells with EDTA to activate Notch1 cleavage. After treatment with $2.5 \mathrm{mM}$ EDTA, the immunofluorescence staining for Notch1 was enriched in the nucleus of HN4 cells (Fig. 1A-d-f). Notch1 cleaved at S2 and S3 sites could be detected by a Notch1-specific antibody (clone D1E11) or Notch1 cleaved at S3 could be directly detected by Notch1 Val1744-specific antibody (clone D3B8). Western blot assays enabled the detection of Notch1 as the expected transmembranous form $(\sim 120 \mathrm{kDa})$ in the HN4 cells (Fig. 1B). A smaller size band expected to be cleaved-NICD was detected after EDTA treatment, but not for the cells treated with $\mathrm{CaCl}_{2}$, which neutralized the function of EDTA (Fig. 1B). These data ascertained the usability of this Notch1 detection method.

Notchl expression in OL and the corresponding OSCC specimens. By performing IHC staining, we assessed the expression of Notch1 in 78 OL specimens and 19 corresponding
OSCC specimens from patients who progressed from OL to OSCC. The weak/moderate/strong levels of Notch1 staining were demonstrated in Fig. 2A-C. In OL, Notch1 was generally expressed in a diffused manner in the suprabasal layer of the epithelium and mostly confined to the nucleus and cytoplasm with variable intensities (Fig. 3A-C). Strong overall expression of Notch1 was observed in $10(13 \%)$ cases (Table I). Notably, in $24(31 \%)$ of the OL samples, membranous staining of Notch1 was observed (Fig. 3D and E, Table I). We noted that lesions with severe dysplasia (Fig. 3D) tended to have stronger membrane staining and cytoplasmic staining of Notch1 than in the lesions with low-to-medium grade dysplasia (Fig. 3E). This membrane staining revealed variable intensity in the spinous layer, granular layer and throughout the whole epithelium compared with the more strictly suprabasal layer expression of Notch1 in the other OL samples.

In the 19 malignant-transformed OL (or OSCC) samples derived from OL cases, 11 (14\%) exhibited positive membranous 

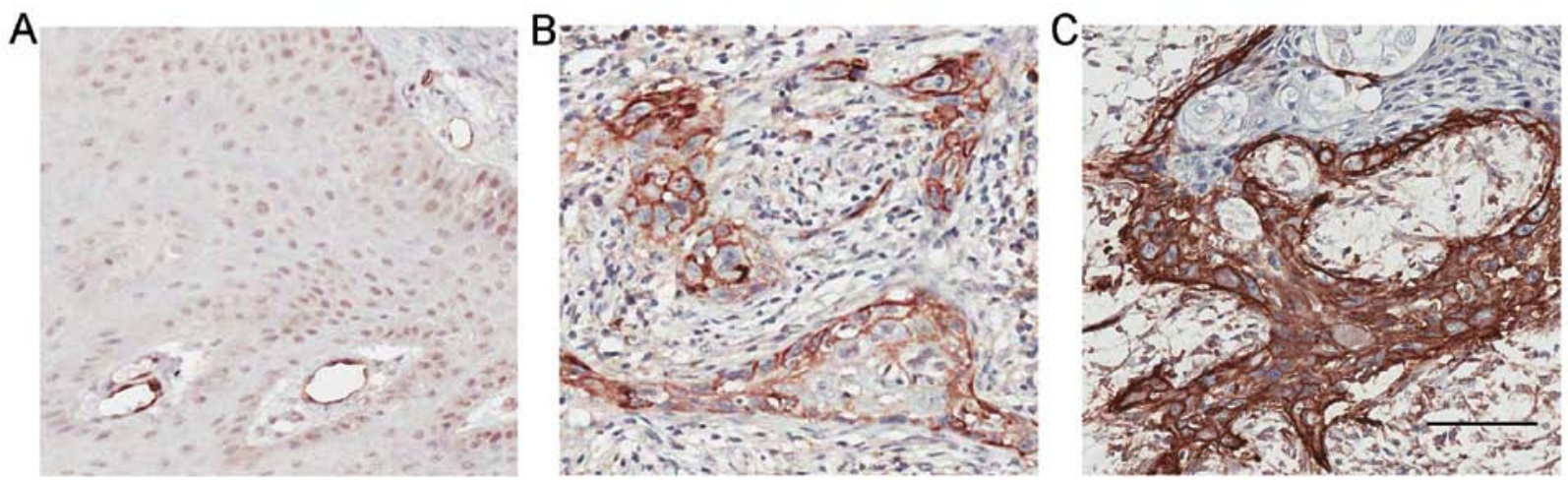

Figure 2. (A-C) The weak/moderate/strong levels of Notch1 staining were demonstrated. Scale bar, $100 \mu \mathrm{m}$.
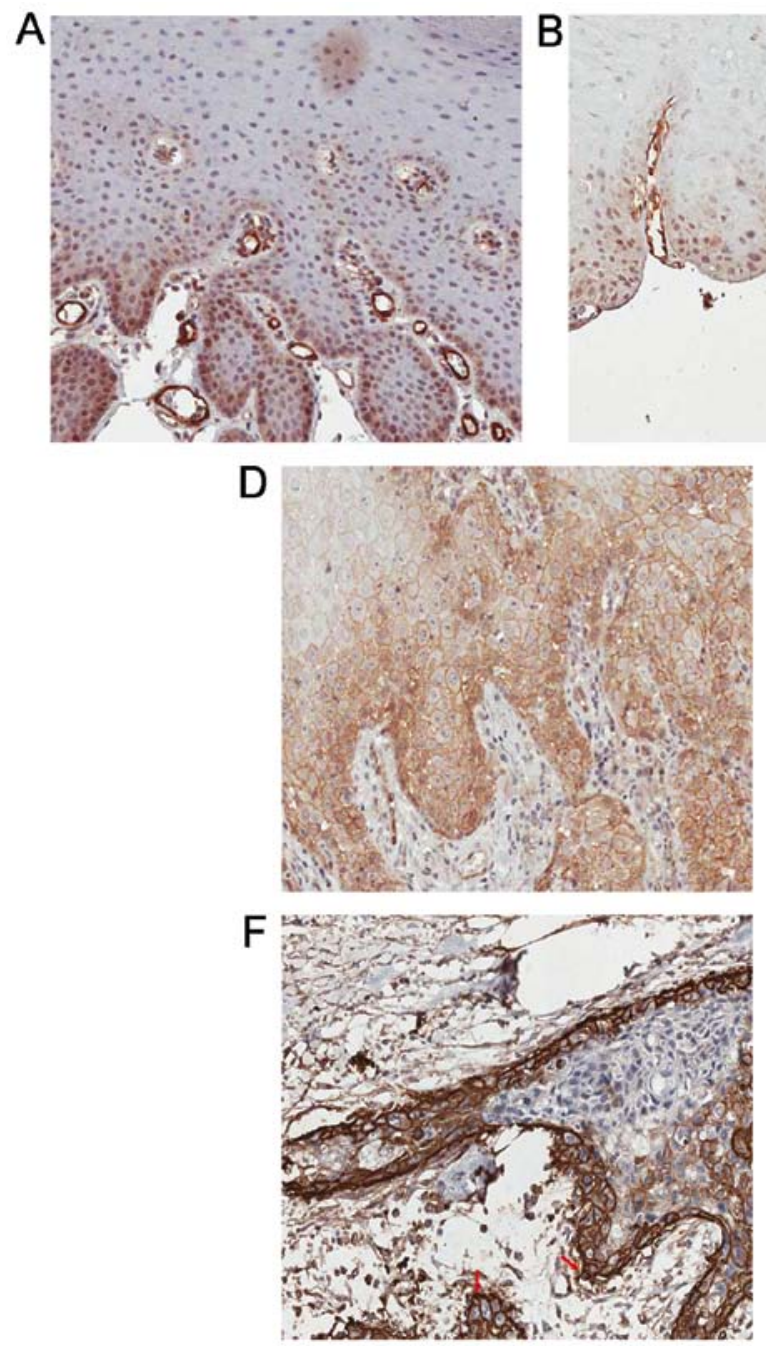
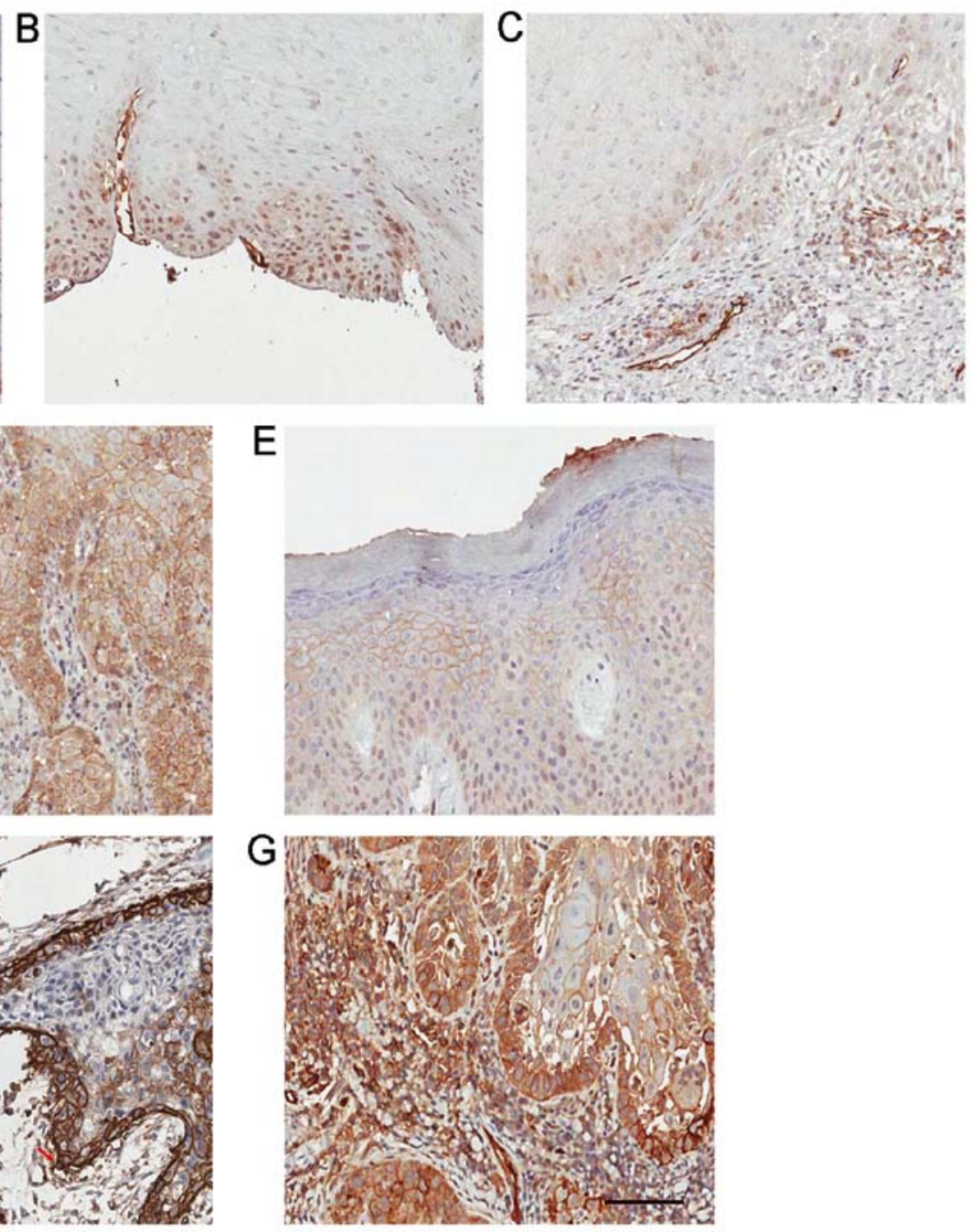

Figure 3. Notch1 expression in OL and the corresponding OSCC specimens. Notch1 was generally expressed in a diffused manner in the suprabasal layer of the epithelium and mostly confined to the nucleus and cytoplasm with variable intensities. (A-C) Representative images of OL specimens with expression of Notch1. (D-G) Membranous expression of Notch1 in OL and their transformed OSCC specimens. Membranous expression of Notch1 in (D) severe dysplasia and in (E) moderate dysplasia. (F) OSCC derived from the same patient as in D. (G) OSCC derived from the same patient as in E. In OSCC samples, Notch1 expression was more diffused on the membrane and in the cytoplasm than in the nucleus. The red arrow indicates strong membrane Notch1 expression at the invasive front or at the border of cancer nests as displayed in F. Scale bar, $100 \mu \mathrm{m}$.

Notch1 expression (Table I). To be specific, in the 19 cases, the lesions in Fig. 3D and $\mathrm{E}$ turned into the malignancies in Fig. 3F and G, respectively during the follow-up studies. Moreover, Notch1 expression was more diffused on the membrane and in the cytoplasm than in the nucleus in these OSCC samples. Strong membranous Notch1 expression was often observed at the invasive front or at the border of cancer nests. Moreover, a significant decreased nuclear 

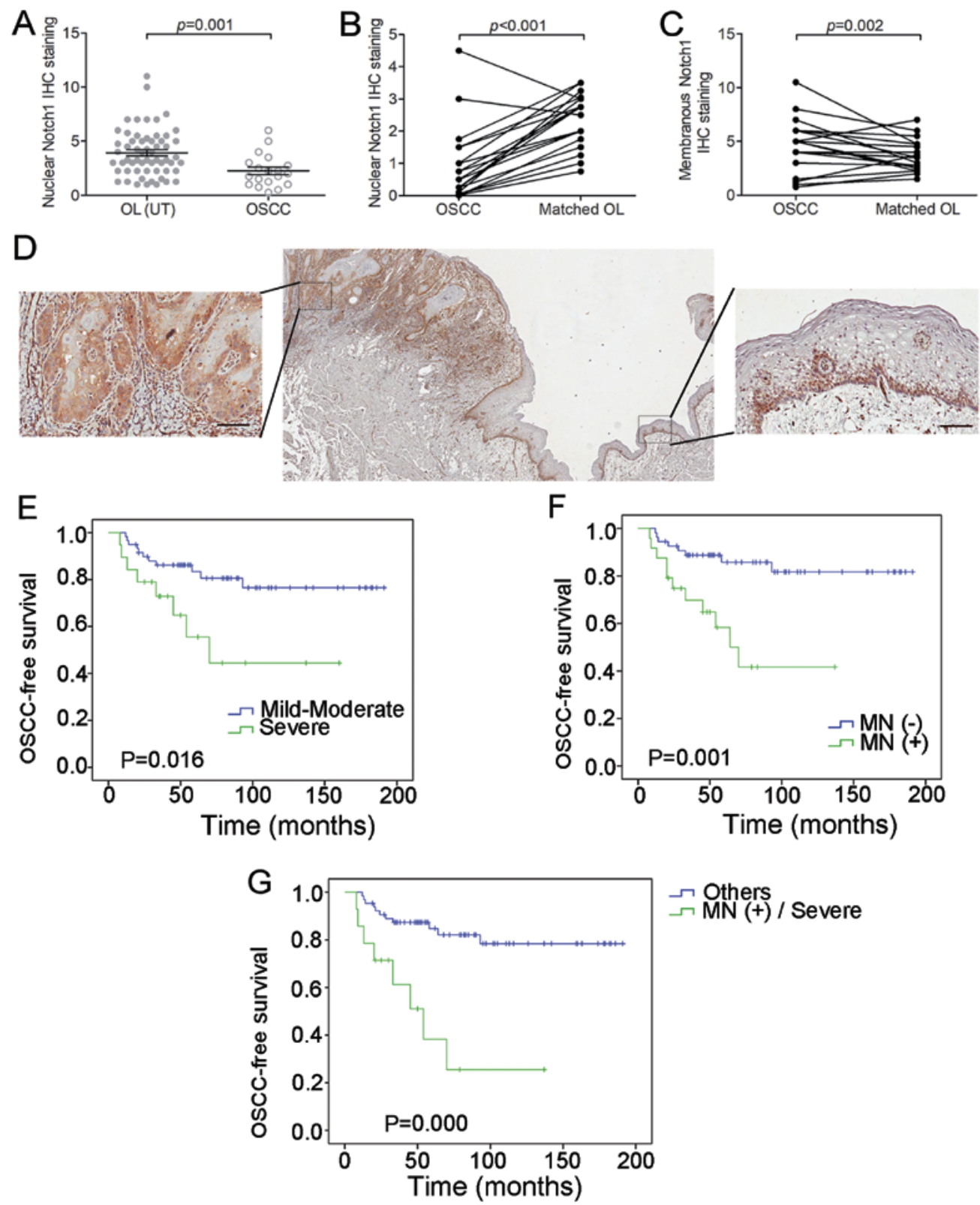

Figure 4. Immunohistochemistry (IHC) staining of membranous and nuclear Notch1 in OL and OSCC specimens. (A) Comparison between 59 untransformed (UT) OL samples and 19 OSCC samples in terms of nuclear Notch1 expression. Nuclear Notch1 expression scores were higher in untransformed (UT) OLs than OSCCs ( $\mathrm{P}=0.001)$. (B) Nuclear Notch1 expression was decreased in OLs compared to their transformed OSCCs $(\mathrm{P}<0.001)$. (C) Membranous Notch1 expression was increased in OLs compared to their transformed OSCCs $(\mathrm{P}=0.002)$. (D) Representative images of samples that revealed decreased expression of nuclear Notch1 and increased expression of membranous Notch1 in cancer nests than in the adjacent non-cancerous epithelium. (E) Association of dysplasia grade with OSCC-free survival ( $\mathrm{P}=0.016)$. (F) Association of membranous Notch1 (MN) with OSCC-free survival ( $\mathrm{P}=0.001)$. (G) Combination of dysplasia grade and MN with OSCC-free survival $(\mathrm{P}=0.000)$. Scale bar, $100 \mu \mathrm{m}$.

Notch1 expression was detected in malignant-transformed (MT) OL samples, compared to the untransformed (UT) OL samples $(\mathrm{P}=0.001$, t-test; Fig. 4A). Meanwhile, a decrease of unclear Notch1 expression $(\mathrm{P}<0.001$, grouped $\mathrm{t}$-test $)$ and an increase of membranous Notch1 expression $(\mathrm{P}=0.002$, grouped t-test) were observed in the 19 OSCC samples compared to their matched OL samples (Fig. 4B and C). Even within the same OSCC sample, we observed a decrease in nuclear Notch1 and an increase in membranous Notch1 expression in cancer nests compared to the adjacent non-cancerous epithelium (Fig. 4D).

Membranous Notchl expression in OL is associated with poor prognosis. The relationship between the membranous Notch1 expression in OL and clinicopathological parameters was also analyzed. A statistically significant association was observed between membranous Notch1 expression and a more severe dysplastic status $(\mathrm{P}<0.001$, Chi-square test, Table II). For further analysis, we determined the role of membranous Notch1 expression in the malignant transformation of OL. As expected, the grade of dysplasia and expression levels of membranous Notch1 were associated with OSCC-free survival $(\mathrm{P}=0.016$ and 0.001, respectively, log-rank test, Fig. 4E and F). After 5 years, only 7 (13\%) of the 54 patients without membranous Notch1 expression developed OSCC, whereas $9(38 \%)$ of the 24 patients with membranous Notch1 expression developed OSCC. When combined with grade of dysplasia, the patients 
Table II. MN with clinicopathological features.

\begin{tabular}{|c|c|c|c|c|c|c|c|}
\hline \multirow[b]{2}{*}{ Characteristic } & \multirow[b]{2}{*}{ No. of patients } & \multirow[b]{2}{*}{$(\%)$} & \multicolumn{2}{|c|}{$\mathrm{MN}(+)$} & \multicolumn{2}{|c|}{ MN (-) } & \multirow[b]{2}{*}{ P-value } \\
\hline & & & $\mathrm{n}$ & $(\%)$ & $\mathrm{n}$ & $(\%)$ & \\
\hline All patients & 78 & 100 & 24 & 31 & 54 & 69 & \\
\hline Age group (years) & & & & & & & 0.462 \\
\hline$<60$ & 54 & 69 & 18 & 23 & 36 & 46 & \\
\hline$\geq 60$ & 24 & 31 & 6 & 8 & 18 & 23 & \\
\hline Sex & & & & & & & 0.567 \\
\hline Female & 45 & 58 & 15 & 19 & 30 & 38 & \\
\hline Male & 33 & 42 & 9 & 12 & 24 & 31 & \\
\hline Grade of dysplasia & & & & & & & $<0.001$ \\
\hline Mild-moderate & 59 & 76 & 11 & 14 & 48 & 61 & \\
\hline Severe & 19 & 24 & 13 & 17 & 6 & 8 & \\
\hline Anatomic site & & & & & & & 0.585 \\
\hline Low-risk areas & 29 & 37 & 10 & 13 & 19 & 24 & \\
\hline High-risk areas & 49 & 63 & 14 & 18 & 35 & 45 & \\
\hline Smoking & & & & & & & 0.95 \\
\hline Yes & 15 & 19 & 4 & 5 & 11 & 14 & \\
\hline No & 57 & 73 & 18 & 23 & 39 & 50 & \\
\hline Unknown & 6 & 8 & 2 & 3 & 4 & 5 & \\
\hline Alcohol drinking & & & & & & & 0.76 \\
\hline Yes & 15 & 19 & 5 & 6 & 10 & 12 & \\
\hline No & 58 & 75 & 17 & 22 & 41 & 53 & \\
\hline Unknown & 5 & 6 & 2 & 3 & 3 & 4 & \\
\hline
\end{tabular}

MN, membrane Notch1 expression.

with membranous Notch1 expression and severe dysplasia exhibited even shorter OSCC-free survival than the other patients $(\mathrm{P}=0.000$, log-rank test, Fig. $4 \mathrm{G})$. As determined by univariate analysis, both the expression level of membranous Notch1 and the grade of dysplasia were associated with OSCC development $(\mathrm{P}=0.002$ and $\mathrm{P}=0.022$, respectively, Table III). By multivariate analysis, however, only membrane Notch1 expression was an independent factor that was significantly associated with OSCC development ( $\mathrm{P}=0.019$, Table III).

Notchl expression patterns in a murine OL/OSCC model. An OL/OSCC model was successfully established in Sprague-Dawley rats by 4-nitroquinoline-1-oxide (4-NQO; Fig. 5A) induction as aforementioned to study the expression of Notch1 in different stages of carcinogenesis. Administration of 4-NQO was performed as outlined in the general scheme in Fig. 5B. We found that the experimental groups (Groups 1-3) treated with 4-NQO developed different pathological changes, including mild dysplasia (Fig. 5C-b), moderate-to-severe dysplasia (Fig. 5C-c) and OSCC (including in situ carcinoma and invasive carcinoma; Fig. 5C-d) with the increase in the duration of 4-NQO administration, and no visible lesions were detected in the control group (Group 4, Fig. 5C-a). The pathological lesions of samples were confirmed by two trained pathologists using H\&E staining in a double-blind fashion (Table IV).
Table III. Univariate and multivariate analysis of clinicopathological features and MN with malignant transformation of OL.

\begin{tabular}{llcc}
\hline Univariate & P-value & Risk ratio & $95 \%$ CI \\
\hline Age (years) & 0.369 & 1.016 & $0.982-1.051$ \\
Sex & 0.062 & 2.665 & $0.952-7.462$ \\
Alcohol intake & 0.74 & 0.871 & $0.384-1.973$ \\
Smoking & 0.301 & 0.611 & $0.241-1.552$ \\
Pathology grade & $\mathbf{0 . 0 2 2}$ & 2.921 & $1.168-7.302$ \\
MN & $\mathbf{0 . 0 0 2}$ & 4.217 & $1.673-10.671$ \\
Multivariate & & & \\
Grade of dysplasia & 0.317 & 1.68 & $0.608-4.643$ \\
MN & $\mathbf{0 . 0 1 9}$ & 3.417 & $1.225-9.529$ \\
\hline
\end{tabular}

MN, membrane Notch1 expression; OL, oral leukoplakia; CI, confidence interval.

The rates of positive expression of Notch1 in normal mucosa, dysplasia and carcinoma were $20 \%$ (2/10), $64.7 \%$ (11/17) and $84.6 \%$ (11/13), respectively ( $\mathrm{P}<0.01$, Chi-square test, Table V). Representative Notch1 IHC images are shown in Fig. 5D. We 


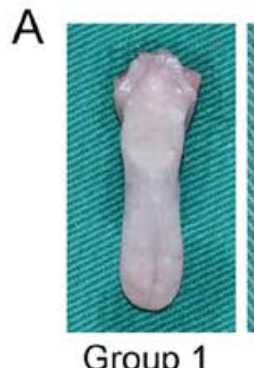

Group 1

(8w)

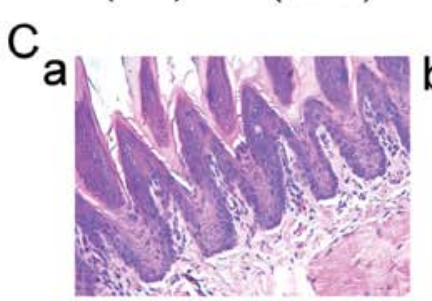

C

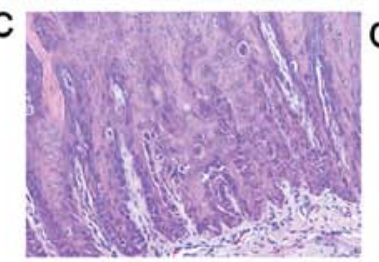

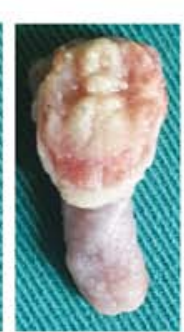

Group 3

(24 w)

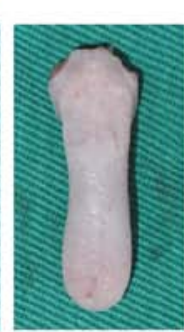

Group 4

(Control)
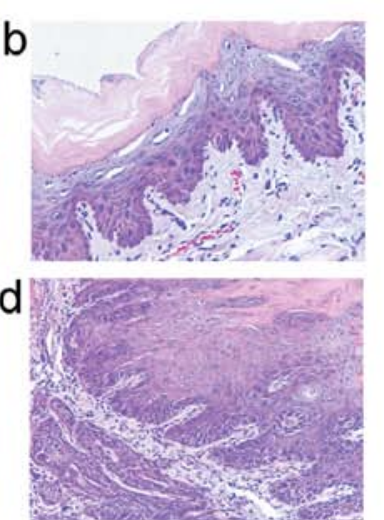

B

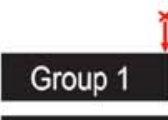

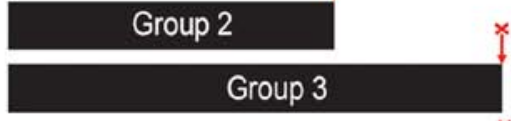

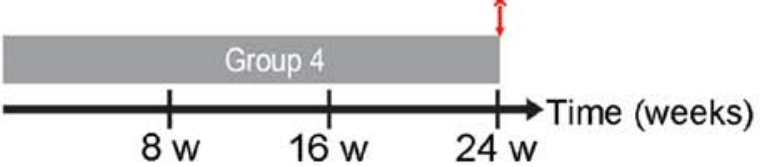

D
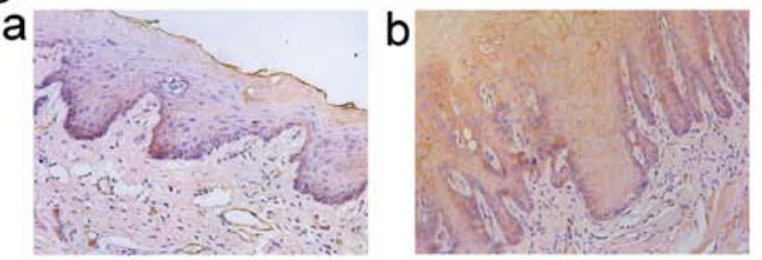

C
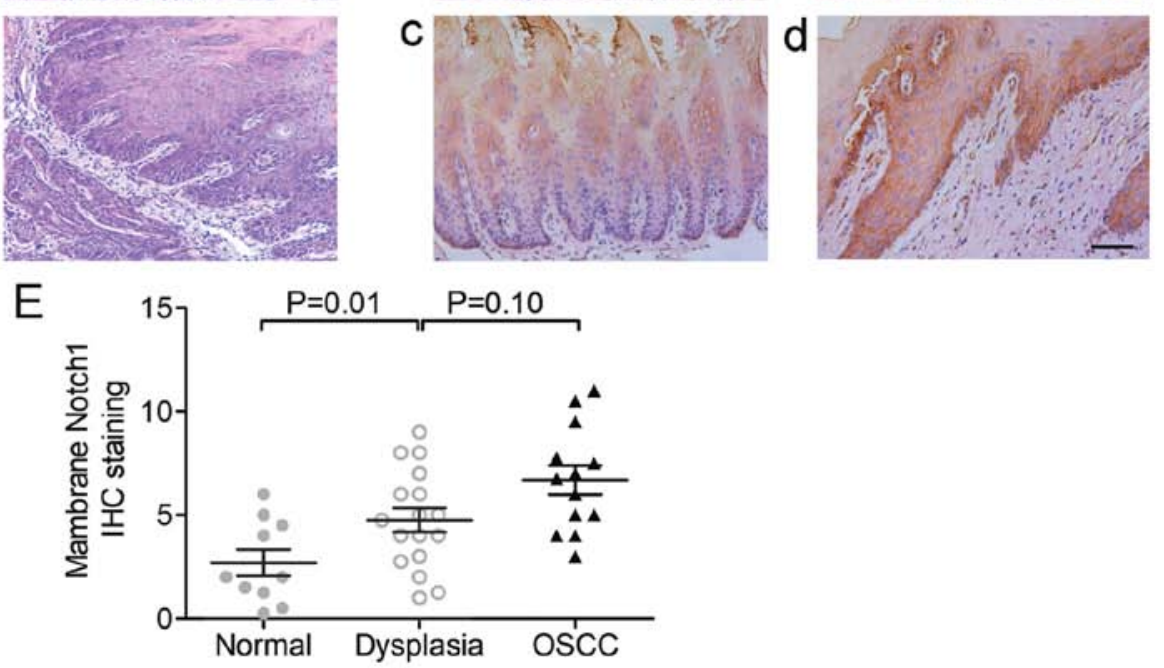

Figure 5. Membranous Notch1 expression is increased during carcinogenesis in a murine OL/OSCC model. (A) Representative images of isolated tongues from SD rats at different administration time-points. (B) General scheme of administration of 4-NQO. (C) Representative images of H\&E staining of tongue tissue samples from SD rats in different stages, including (a) normal epithelium, (b) mild dysplasia, (c) moderate-to-severe dysplasia and (d) OSCC. (D) Representative images of Notch1 IHC staining. As shown, Notch1 was mainly localized in the membrane and cytoplasm in the murine tongue tissues. Notch1 staining was negative in the normal tongue mucosa or was mainly distributed in the stratum basale (a). It extended from the stratum basale to the stratum corneum during the progression of cancer (a-d). (E) The scores of membranous Notch1 expression in different stages during carcinogenesis was analyzed. Scale bar, $100 \mu$ m.

Table IV. Pathological classification of tongue tissues at different administration times.

\begin{tabular}{lcccccc}
\hline & & \multicolumn{5}{c}{ Pathological lesions } \\
\cline { 3 - 6 } Groups & No. & Normal epithelial & $\begin{array}{c}\text { Mild epithelial } \\
\text { dysplasia }\end{array}$ & $\begin{array}{c}\text { Moderate-severe } \\
\text { epithelial dysplasia }\end{array}$ & In situ carcinoma & $\begin{array}{c}\text { Invasive } \\
\text { carcinoma }\end{array}$ \\
\hline 1 (8 weeks) & 10 & 0 & 7 & 3 & 0 & 0 \\
2 (16 weeks) & 10 & 0 & 2 & 4 & 3 & 1 \\
3 (24 weeks) & 10 & 0 & 0 & 1 & 6 & 3 \\
4 (Control) & 10 & 10 & 0 & 0 & 9 & 0 \\
Total & 40 & 10 & 9 & 8 & 9 \\
\hline
\end{tabular}

found that Notch1 was mainly localized in the membrane and cytoplasm in this murine model. Notch1 staining was negative in the normal tongue mucosa or was mainly distributed in the stratum basale (Fig. 5D-a), whereas it extended from the stratum basale to the stratum corneum during the progression of cancer (Fig. 5D-a-d). The IHC scores of membranous Notch1 expression were also increased during carcinogenesis (Fig. 5E). 
Table V. Expression of Notch1 in normal mucosa and different stages of carcinogenesis.

\begin{tabular}{lccc}
\hline Pathological lesions & No. & Negative & Positive \\
\hline Normal & 10 & 8 & 2 \\
Dysplasia & 17 & 6 & 11 \\
OSCC & 13 & 2 & 11 \\
\hline
\end{tabular}

OSCC, oral squamous cell carcinoma.

\section{Discussion}

Notch1 signaling has been studied in various types of malignancies. Although Notch1 signaling has been demonstrated to play a significant oncogenic role in T-ALL (32), its role in several solid tumors, including OSCC and HNSCC, remains controversial even within the same tumor type $(32,33)$. For example, both increased (18,34-37) and decreased Notch1 expression or Notch1 signaling has been discovered in OSCC and HNSCC samples, and improved (38) or worsened (37) survival has been revealed in HNSCC. The reason behind these discrepancies may be due to the cellular context, since the Notch signaling pathway can play opposing roles in malignancies, depending on the cellular and tissue context, as well as the level of its expression and potential crosstalk with other signaling pathways (39).

However, the detection methods for Notch1 have not been convincing, as very few studies have validated the Notch1-specific antibodies before using them, and this could have also contributed to the discrepancies. In the present study, we first validated the Notch1-specific antibody using several protocols to ensure that it could detect both the membrane-bound and nuclear Notch1. First, membranous, cytoplasmic and nuclear Notch1 staining was detected using the Notch1 primary antibody by immunofluorescence. Calcium depletion by EDTA from the medium triggered a ligand-independent activation of Notch signaling and nuclear Notch1 could be detected by the antibody using both western blotting and immunofluorescence; meanwhile, treatment with $\mathrm{CaCl}_{2}$ neutralized the function of EDTA and reversed the effect.

Subsequently, we provided an extensive evaluation of Notch1 expression in human OL and OSCC tissue samples. We determined that in our OL samples, Notch1 was mainly localized in the suprabasal layer of the epithelium, indicating its role in cell differentiation. This result was consistent with previous studies which demonstrated that in normal oral epithelium, Notch was mainly expressed in the suprabasal layer $(36,40)$. Although a previous study (36) mentioned that nuclear Notch1 could hardly be detected in normal tissues, we found strong nuclear expression of Notch1 in some OL or OSCC tissues, and the nuclear expression of Notch1 was decreased during the progression from OL to OSCC. Moreover, within the same OSCC sample, we noticed a decrease in nuclear Notch1 from the peri-cancerous epithelium to the cancer nests, revealing that canonical Notch signaling plays a tumor-suppressive role in normal oral epithelium and OL. In fact, there have been studies on nuclear Notch1 expression in variable tissues during development and in cancer, although the exact role of nuclear Notch1 is debatable (41-43).

We also found diffused membranous Notch1 expression in 24 OL samples, which had not been studied in detail before. In the present study, membranous Notch1 expression was significantly associated with advanced dysplasia and malignant transformation. Moreover, membranous Notch1 expression was significantly higher in the OSCC samples than in the OL samples. Notably, the elevated membranous expression in OSCC was mainly observed at the border of cancer nests or at invasive fronts. According to the Cox proportional hazards regression model, the presence of membranous Notch1 was even more specific and independent than the pathological grade for predicting malignant transformation in OL. In our murine model induced by 4-NQO gavages, similar Notch1 expression patterns were also detected. Notch1 staining extended from the stratum basale to the stratum corneum during the development of cancer. The rate of membranous Notch1 expression also increased during carcinogenesis.

It should be noted that NOTCH1 mutation frequency was as high as $60 \%$ in OL, and almost $60 \%$ of leukoplakia patients with mutations were identified in OSCC, although these OL and OSCC samples were not from the same patient (44). Moreover, these mutations were mostly confined to the EGF-like domain, which accounts for ligand-receptor binding. An impaired ligand-receptor binding resulting from a mutation in this domain of Notch1 may theoretically cause a surplus of Notch1 proteins and thus passively result in its accumulation on the membrane. Therefore, it is important to determine whether the $\sim 30 \%$ membranous Notch1 expression revealed in our research is a reflection of some of the common mutations found both in OL and in OSCC. It should be noted, however, that the study described by Izumchenko et al (44) and the present study were based on Chinese populations. Potential impact of etiology and genetic background for the particular population should be considered in the data interpretation. As Notch1 has been suggested as a tumor suppressor in several studies $(23,45,46)$, it is possible that membranous Notch1 accumulation is simply an alternative mechanism to inactivate the gene function. However, a recent study revealed that the Notch signaling pathway was active in about one third of Caucasian patients with HNSCC (34), suggesting an oncogenic role of Notch signaling in a significant proportion of patients with HNSCC in both Chinese and Caucasian populations, although the mechanisms of tumorigenesis could be different (47). Further studies are warranted to determine how membranous Notch1 accumulation leads to oral tumorigenesis and OSCC progression.

\section{Acknowledgements}

Not applicable.

\section{Funding}

The present study was supported by the National Natural Science Foundation of China (no. 81402236), the National Natural Science Foundation of China (no. 81772887), 
the Priority Academic Program Development of Jiangsu Higher Education Institutions (PAPD, 2014-37), the Jiangsu Provincial Medical Innovation Team (CXTDA2017036), the Natural Science Foundation of Jiangsu Province of China (BK20171488) and the Jiangsu Provincial Medical Youth Talent (QNRC2016854).

\section{Availability of data and materials}

The datasets used during the present study are available from the corresponding author upon reasonable request.

\section{Authors' contributions}

$\mathrm{XD}, \mathrm{YZ}$ and $\mathrm{YW}$ conceived and designed the study. XD, YZ, ZW, YD and WZ performed the experiments. YZ, ZY, WZ and $\mathrm{XS}$ wrote the manuscript. WC, JL, WC, LM and WZ reviewed and edited the manuscript. All authors read and approved the manuscript and agree to be accountable for all aspects of the research in ensuring that the accuracy or integrity of any part of the work are appropriately investigated and resolved.

\section{Ethics approval and consent to participate}

The research was carried out with the approval from the Ethics Committee of Shanghai Jiao Tong University (Shanghai, China).

\section{Consent for publication}

Not applicable.

\section{Competing interests}

The authors declare that they have no conflict of interest.

\section{References}

1. Lodi G, Sardella A, Bez C, Demarosi F and Carrassi A: Interventions for treating oral leukoplakia. Cochrane Database Syst Rev 18: CD001829, 2006.

2. Silverman S Jr, Gorsky M and Lozada F: Oral leukoplakia and malignant transformation. A follow-up study of 257 patients. Cancer 53: 563-568, 1984.

3. van der Waal I: Potentially malignant disorders of the oral and oropharyngeal mucosa; terminology, classification and present concepts of management. Oral Oncol 45: 317-323, 2009.

4. Papadimitrakopoulou VA, Hong WK, Lee JS, Martin JW, Lee JJ, Batsakis JG and Lippman SM: Low-dose isotretinoin versus beta-carotene to prevent oral carcinogenesis: Long-term follow-up. J Natl Cancer Inst 89: 257-258, 1997.

5. Schepman KP, van der Meij EH, Smeele LE and van der Waal I: Malignant transformation of oral leukoplakia: A follow-up study of a hospital-based population of 166 patients with oral leukoplakia from The Netherlands. Oral Oncol 34: 270-275, 1998.

6. Yuan P, Temam S, El-Naggar A, Zhou X, Liu DD, Lee JJ and Mao L: Overexpression of podoplanin in oral cancer and its association with poor clinical outcome. Cancer 107: 563-569, 2006.

7. Kawaguchi H, El-Naggar AK, Papadimitrakopoulou V, Ren H, Fan YH, Feng L, Lee JJ, Kim E, Hong WK, Lippman SM and Mao L: Podoplanin: A novel marker for oral cancer risk in patients with oral premalignancy. J Clin Oncol 26: 354-360, 2008.

8. Mitra D, Fernandez P, Bian L, Song N, Li F, Han G and Wang XJ: Smad4 loss in mouse keratinocytes leads to increased susceptibility to UV carcinogenesis with reduced ercc1-mediated DNA repair. J Invest Dermatol 133: 2609-2616, 2013.
9. Xia RH, Song XM, Wang XJ, Li J and Mao L: The combination of SMAD4 expression and histological grade of dysplasia is a better predictor for the malignant transformation of oral leukoplakia. PLoS One 8: e66794, 2013.

10. Kopan R and Ilagan MX: The canonical Notch signaling pathway: Unfolding the activation mechanism. Cell 137: 216-233, 2009.

11. Guruharsha KG, Kankel MW and Artavanis-Tsakonas S: The Notch signalling system: Recent insights into the complexity of a conserved pathway. Nat Rev Genet 13: 654-666, 2012.

12. Zheng Y, Wang Z, Ding X, Dong Y, Zhang W, Zhang W, Zhong Y, Gu W, Wu Y and Song X: Combined Erlotinib and PF-03084014 treatment contributes to synthetic lethality in head and neck squamous cell carcinoma. Cell Prolif: Dec 12, 2017 (Epub ahead of print).

13. Ranganathan P, Weaver KL and Capobianco AJ: Notch signalling in solid tumours: A little bit of everything but not all the time. Nat Rev Cancer 11: 338-351, 2011.

14. Patturajan M, Nomoto S, Sommer M, Fomenkov A, Hibi K, Zangen R, Poliak N, Califano J, Trink B and Ratovitski E: DeltaNp63 induces beta-catenin nuclear accumulation and signaling. Cancer Cell 1: 369-379, 2002.

15. Sriuranpong V, Borges MW, Ravi RK, Arnold DR, Nelkin BD, Baylin SB and Ball DW: Notch signaling induces cell cycle arrest in small cell lung cancer cells. Cancer Res 61: 3200-3205, 2001.

16. Yugawa T, Handa K, Narisawa-Saito M, Ohno S, Fujita M and Kiyono T: Regulation of Notch1 gene expression by p53 in epithelial cells. Mol Cell Biol 27: 3732-3742, 2007.

17. Panelos J, Tarantini F, Paglierani M, Di Serio C, Maio V, Pellerito S, Pimpinelli N, Santucci M and Massi D: Photoexposition discriminates Notch 1 expression in human cutaneous squamous cell carcinoma. Mod Pathol 21: 316-325, 2008.

18. Zhang TH, Liu HC, Zhu LJ, Chu M, Liang YJ, Liang LZ and Liao GQ: Activation of Notch signaling in human tongue carcinoma. J Oral Pathol Med 40: 37-45, 2011

19. Yao J, Duan L, Fan M and Wu X: Gamma secretase inhibitors exerts antitumor activity via down-regulation of Notch and Nuclear factor kappa B in human tongue carcinoma cells. Oral Dis 13: 555-563, 2007.

20. Lin JT, Chen MK, Yeh KT, Chang CS, Chang TH, Lin CY, Wu YC, Su BW, Lee KD and Chang PJ: Association of high levels of Jagged-1 and Notch-1 expression with poor prognosis in head and neck cancer. Ann Surg Oncol 17: 2976-2983, 2010.

21. Hijioka H, Setoguchi T, Miyawaki A, Gao H, Ishida T, Komiya S and Nakamura N: Upregulation of Notch pathway molecules in oral squamous cell carcinoma. Int J Oncol 36: 817-822, 2010.

22. Duan L, Yao J, Wu X and Fan M: Growth suppression induced by Notch1 activation involves Wnt-beta-catenin down-regulation in human tongue carcinoma cells. Biol Cell 98: 479-490, 2006.

23. Stransky N, Egloff AM, Tward AD, Kostic AD, Cibulskis K, Sivachenko A, Kryukov GV, Lawrence MS, Sougnez C, McKenna A, et al: The mutational landscape of head and neck squamous cell carcinoma. Science 333: 1157-1160, 2011.

24. Agrawal N, Frederick MJ, Pickering CR, Bettegowda C, Chang K, Li RJ, Fakhry C, Xie TX, Zhang J, Wang J, et al: Exome sequencing of head and neck squamous cell carcinoma reveals inactivating mutations in Notch1. Science 333: 1154-1157, 2011.

25. Song X, Xia R, Li J, Long Z, Ren H, Chen W and Mao L: Common and complex Notch1 mutations in chinese oral squamous cell carcinoma. Clin Cancer Res 20: 701-710, 2014.

26. Izumchenko E, Sun K, Jones S, Brait M, Agrawal N, Koch WM, McCord C, Riley D, Angiuoli SV, Velculescu VE, et al: Notch1 mutations are drivers of oral tumorigenesis. Cancer Prev Res 8: 277-286, 2014.

27. Cao W, Feng Z, Cui Z, Zhang C, Sun Z, Mao L and Chen W: Up-regulation of enhancer of zeste homolog 2 is associated positively with cyclin D1 overexpression and poor clinical outcome in head and neck squamous cell carcinoma. Cancer 118: 2858-2871, 2011.

28. Xu W,Liang CG, Li YF, Ji YH, Qiu WJ and Tang XZ: Involvement of Notch1/Hes signaling pathway in ankylosing spondylitis. Int J Clin Exp Pathol 8: 2737-2745, 2015.

29. Lakhani SR, Van De Vijver MJ, Jacquemier J, Anderson TJ, Osin PP, Mcguffog L and Easton DF: The pathology of familial breast cancer: Predictive value of immunohistochemical markers estrogen receptor, progesterone receptor, HER-2, and p53 in patients with mutations in BRCA1 and BRCA2. J Clin Oncol 20: 2310-2328, 2002. 
30. Hasina R, Martin LE, Kasza K, Jones CL, Jalil A and Lingen MW: ABT-510 is an effective chemopreventive agent in the mouse 4-nitroquinoline 1-oxide model of oral carcinogenesis. Cancer Prev Res 2: 385-393, 2009.

31. Chang NW, Pei RJ, Tseng HC, Yeh KT, Chan HC, Lee MR, Lin C, Hsieh WT, Kao MC, Tsai MH and Lin CF: Co-treating with arecoline and 4-nitroquinoline 1-oxide to establish a mouse model mimicking oral tumorigenesis. Chem Biol Int 183: 231-237, 2010

32. South AP, Cho RJ and Aster JC: The double-edged sword of Notch signaling in cancer. Semin Cell Dev Biol 23: 458-464, 2012.

33. Lefort K, Ostano P, Mello-Grand M, Calpini V, Scatolini M, Farsetti A, Dotto GP and Chiorino G: Dual tumor suppressing and promoting function of Notch1 signaling in human prostate cancer. Oncotarget 7: 48011-48026, 2016.

34. Sun W, Gaykalova DA, Ochs MF, Mambo E, Arnaoutakis D, Liu Y, Loyo M, Agrawal N, Howard J, Li R, et al: Activation of the Notch pathway in head and neck cancer. Cancer Rese 74 1091-1104, 2014

35. Upadhyay P, Nair S, Kaur E, Aich J, Dani P, Sethunath V, Gardi N, Chandrani P, Godbole M, Sonawane K, et al: Notch pathway activation is essential for maintenance of stem-like cells in early tongue cancer. Oncotarget 7: 50437-504449, 2016.

36. Yoshida R, Nagata M, Nakayama H, Niimori-Kita K, Hassan W, Tanaka T, Shinohara M and Ito T: The pathological significance of Notch1 in oral squamous cell carcinoma. Lab Invest 93: 1068-1081, 2013

37. Lee SH, Do SI, Lee HJ, Kang HJ, Koo BS and Lim YC: Notch1 signaling contributes to stemness in head and neck squamous cell carcinoma. Lab Invest 96: 508-516, 2016 (In English).

38. Kaka AS, Nowacki NB, Kumar B, Zhao S, Old MO, Agrawal A Ozer E, Carrau RL, Schuller DE, Kumar P and Teknos TN: Notch1 overexpression correlates to improved survival in cancer of the oropharynx. Otolaryngol Head Neck Surg 156: 652-659, 2017.

39. Miele L: Notch signaling. Clin Cancer Res 12: 1074-1079, 2006.
40. Sakamoto K, Fujii T, Kawachi H, Miki Y, Omura K, Morita K, Kayamori K, Katsube K and Yamaguchi A: Reduction of Notch1 expression pertains to maturation abnormalities of keratinocytes in squamous neoplasms. Lab Invest 92: 688-702, 2012.

41. Artavanis-Tsakonas S, Rand MD and Lake RJ: Notch signaling: Cell fate control and signal integration in development. Science 284: 770-776, 1999.

42. Zagouras P, Stifani S, Blaumueller CM, Carcangiu ML and Artavanis-Tsakonas S: Alterations in Notch signaling in neoplastic lesions of the human cervix. Proc Natl Acad Sci USA 92: 6414-6418, 1995

43. Ahmad I, Zaqouras P and Artavanis-Tsakonas S: Involvement of Notch-1 in mammalian retinal neurogenesis: Association of Notch-1 activity with both immature and terminally differentiated cells. Mech Dev 53: 73-85, 1995.

44. Izumchenko E, Sun K, Jones S, Brait M, Agrawal N, Koch W, McCord CL, Riley DR, Angiuoli SV, Velculescu VE, et al: Notch1 mutations are drivers of oral tumorigenesis. Cancer Prev Res 8: 277-286, 2015.

45. Zweidler-Mckay PA, He Y, Xu L, Rodriguez CG, Karnell FG, Carpenter AC, Aster JC, Allman D and Pear WS: Notch signaling is a potent inducer of growth arrest and apoptosis in a wide range of B-cell malignancies. Blood 106: 3898-3906, 2005.

46. Nicolas M, Wolfer A, Raj K, Kummer JA, Mill P, van Noort M, Hui CC, Clevers H, Dotto GP and Radtke F: Notch1 functions as a tumor suppressor in mouse skin. Nat Genet 33: 416-421, 2003.

47. Zheng Y, Wang Z, Ding X, Zhang W, Li G, Liu L, Wu H, Gu W, Wu Y and Song X: A novel Notch1 missense mutation (C1133Y) in the Abruptex domain exhibits enhanced proliferation and invasion in oral squamous cell carcinoma. Cancer Cell Int 18: 6, 2018.

(i) $\Theta$ This work is licensed under a Creative Commons Attribution-NonCommercial-NoDerivatives 4.0 International (CC BY-NC-ND 4.0) License. 